Homology, Homotopy and Applications, vol.10(3), 2008, pp.181-192

\title{
THE BP-THEORY OF 2-FOLD PRODUCTS OF REAL PROJECTIVE SPACES
}

\author{
JESÚS GONZÁLEZ AND W. STEPHEN WILSON
}

(communicated by Donald M. Davis)

\begin{abstract}
We study the Brown-Peterson (co)homology of a product of two real projective spaces via the Landweber short exact sequence. The image of the tensor product is well understood. Our contribution is to understand those elements not in the tensor product and to show how they behave under maps. The results are partially extended to the case where one of the factors is replaced by a $2^{e}$-torsion lens space.
\end{abstract}

\section{Introduction}

In $[\mathbf{K W a}, \mathbf{K W} \mathbf{W}]$, the need for the Brown-Peterson cohomology (for $p=2$ ) of a product of two real projective spaces arose. In particular, they needed to understand the elements not in the tensor product and how they behaved under maps.

Although quick computations with the Adams spectral sequence or the AtiyahHirzebruch spectral sequence suggest the answer, there seemed to be nothing explicit enough in the literature, but much of what we do is well known.

Unless otherwise stated, we use reduced cohomology. Recall $B P^{*} \approx \mathbb{Z}_{(2)}\left[v_{1}, v_{2}, \ldots\right]$. Let $x \in B P^{2}\left(R P^{2 k}\right)$ denote the standard generator coming from $B P^{*}\left(C P^{\infty}\right)$. The required theorems are as follows:

Theorem 1.1. Let $m \leqslant n$; then (in reduced cohomology)

$$
\begin{gathered}
B P^{*}\left(R P^{2 m} \wedge R P^{2 n}\right) \approx B P^{*}\left(R P^{2 m}\right) \otimes_{B P^{*}} B P^{*}\left(R P^{2 n}\right) \oplus \Sigma^{2 n-1} B P^{*}\left(R P^{2 m}\right) \\
B P^{*}\left(R P^{2 m} \wedge R P^{2 n+1}\right) \approx B P^{*}\left(R P^{2 m} \wedge R P^{2 n}\right) \oplus \Sigma^{2 n+1} B P^{*}\left(R P^{2 m}\right) .
\end{gathered}
$$

Both isomorphisms can be chosen compatible with maps induced by inclusions $R P^{2 m-2 k} \rightarrow R P^{2 m}$ on the first smash-factor.

All of the groups are finite 2-torsion. A 2-adic basis for $B P^{*}\left(R P^{2 k}\right)$ is given by all $v x^{i}$ with $0<i \leqslant k$, and where $v$ ranges over a $\mathbb{Z}_{(2)}$-basis of $B P^{*}$.

$A$ 2-adic basis for $B P^{*}\left(R P^{2 m}\right) \otimes_{B P^{*}} B P^{*}\left(R P^{2 n}\right)$ is given by all vx $x^{i} x^{j}$ with $0<i \leqslant m, 0<j \leqslant n$, and where $v$ ranges over $a \mathbb{Z}_{(2)}$-basis of $B P^{*}$ when $j=1$ and over a $\mathbb{Z}_{(2)}$-basis of $\mathbb{Z}_{(2)}\left[v_{2}, v_{3}, \ldots\right]$ when $j>1$.

Received August 20, 2007, revised April 7, 2008; published on December 5, 2008.

2000 Mathematics Subject Classification: 55N22, 55R40.

Key words and phrases: Brown-Peterson homology, real projective spaces.

This article is available at http://intlpress.com/HHA/v10/n3/a9

Copyright (c) 2008, International Press. Permission to copy for private use granted. 
A variety of comments are in order here. A 2-adic basis of a finite (graded) abelian group $G$ is a set $\beta \subset G$ such that every element of $G$ can be written as a unique linear combination of the elements of $\beta$ using only coefficients 0 and 1 . In some cases 2 -adic bases turn out to be closely related to bases of the graded object associated to a filtration of $G$ for which this associated object happened to be a $\mathbb{Z} /(2)$-vector space ${ }^{1}$. In our context, the standard example comes from the usual (unreduced) expression

$$
B P^{*}\left(R P^{2 k}\right) \approx B P^{*}[x] /\left(x^{k+1}, 2 x+a_{1} x^{2}+a_{2} x^{3}+\cdots\right), \quad a_{i} \in B P^{*} .
$$

The associated graded object $A=\left\{F_{a} / F_{a+1}\right\}_{a \geqslant 0}$ with respect to the multiplicative decreasing filtration determined by $F(x)=1$ becomes a $\mathbb{Z} /(2)$-vector space if we only care about the reduced part of $B P^{*}\left(R P^{2 k}\right)$. The set of all classes $\widetilde{b} \in A_{F(b)}$, with $b$ ranging over the first 2-adic basis in Theorem 1.1, is a $\mathbb{Z} /(2)$-vector space basis of $A$. Considering 2 -adic bases gives a clean way for avoiding dealing with group extension intricacies coming from the main relation $0=2 x+a_{1} x^{2}+a_{2} x^{3}+\cdots$. This has an even more dramatic (but simplifying) effect when considering the tensor product $B P^{*}\left(R P^{2 m}\right) \otimes_{B P^{*}} B P^{*}\left(R P^{2 n}\right)$. In fact, our description manages to avoid the hard analysis in [Dav84] of the latter group structure, and yet to come up with an answer useful for the geometric goals in $[\mathbf{K W a}, \mathbf{K W b}]$.

The first isomorphism in Theorem 1.1 is in fact functorial with respect to inclusions on the first smash-factor. However this is not quite true for the second isomorphism, as it is obtained from choosing explicit splittings of $B P \wedge R P_{b}^{a}$ for odd $a$ or even $b$. $\left(R P_{b}^{a}\right.$ is the cofiber of the map $R P^{b-1} \rightarrow R P^{a}$.) It is certainly possible to describe the maps induced by inclusions $R P^{2 m-2 k} \rightarrow R P^{2 m}$ on each of the summands in the isomorphisms in Theorem 1.1. Due to its relevance for $[\mathbf{K W a}, \mathbf{K W b}]$, this is carefully indicated in Theorem 1.2 below for the (suspended) summand of the first isomorphism of Theorem 1.1.

We can, of course, handle the $B P$ (co)homology of any $R P_{b}^{a} \wedge R P_{d}^{c}$ for any $a>b$ and $c>d$. What we state in the above theorem is precisely what is needed in [KWa, $\mathbf{K W b}$. We actually go much further and look at the situation when one of the spaces is a $2^{r}$ lens space.

Some of the hard work here was done long ago by Conner and Floyd in Chapter 8 of [CF64] where they computed the tensor product part of $M S O_{*}(B \mathbb{Z} /(p) \times B \mathbb{Z} /(p))$. They didn't have $B P$, and $M U$ wasn't in common usage yet, so their work is at odd primes, but it shows the way.

There is more to this than just the tensor product. Peter Landweber set up a general short exact sequence in [Lan66] that gives, among other things,

$$
B P_{*}(X) \otimes_{B P_{*}} B P_{*}(Y) \longrightarrow B P_{*}(X \wedge Y) \longrightarrow \operatorname{Tor}^{B P_{*}}\left(B P_{*}(X), B P_{*}(Y)\right)
$$

when $X$ is such that $B P_{*}(X)$ surjects to $H_{*}(X)$, in particular, when $X$ is $R P^{n}$ or a lens space. Our main contribution to the above theorem is to make the Tor term explicit algebraically (there is no topology involved) and to show how it behaves under the map we describe. In particular, we show:

\footnotetext{
${ }^{1}$ With the exception of the $B P$-theory of a single lens space of torsion $2^{e}, e>1$, discussed in Theorem 1.5, this is the case for all situations considered in this paper.
} 
Theorem 1.2. Let $m \leqslant n$; then

$$
\operatorname{Tor}^{B P^{*}}\left(B P^{*}\left(R P^{2 m}\right), B P^{*}\left(R P^{2 n}\right)\right) \approx \Sigma^{2 n} B P^{*}\left(R P^{2 m}\right) .
$$

This isomorphism is compatible with the inclusion map $R P^{2 m-2 k} \rightarrow R P^{2 m}$. On the other hand, if $m<n$, the map $R P^{2 n-2} \rightarrow R P^{2 n}$ induces the map

$$
\Sigma^{2 n} B P^{*}\left(R P^{2 m}\right) \longrightarrow \Sigma^{2 n-2} B P^{*}\left(R P^{2 m}\right)
$$

that takes $x^{i}$ to $x^{i+1}$.

Note that the map to Tor in the cohomology Landweber short exact sequence raises degree by 1 .

Note that to compute what happens on the Tor term for a map of $R P^{2 k} \rightarrow R P^{2 n}$ with $k<m<n$ we use the composition $R P^{2 k} \rightarrow R P^{2 m} \rightarrow R P^{2 n}$ and use the second form of the mapping for $R P^{2 m} \rightarrow R P^{2 n}$ and the first for the $R P^{2 k} \rightarrow R P^{2 m}$.

In [JW 85], where a lot of work similar to this is done, credit is given to Bob Stong for knowing the Tor term when both $n$ and $m$ are infinity in the homology case, so even this is not entirely new.

However, the applications in $[\mathbf{K W a}, \mathbf{K W} \mathbf{b}]$ are significant and are used to give new non-immersions of real projective spaces in fairly low dimensions. Since we could find nothing like the above theorem in the literature we felt it necessary to write this up to support the applications.

In the first part of Theorem 1.1, the two parts coming from the Landweber short exact sequence are even and odd degree so there can be no extension problems to consider. In the second part there could be, and when we look at the general case of $R P_{b}^{a} \wedge R P_{d}^{c}$ for any $a>b$ and $c>d$ there could, in principle, be several possible extension problems. None of these occur. This next result tells how to compute the $B P$ cohomology of all such products by combining several known facts. Recall that since we are using reduced cohomology, $B P^{*}\left(R P^{n}\right)=B P^{*}\left(R P_{1}^{n}\right)$.

\section{Theorem 1.3.}

$B P^{*}\left(R P_{2 b+1}^{2 a}\right) \approx \Sigma^{2 b} B P^{*}\left(R P^{2(a-b)}\right)$.

$B P^{*}\left(R P_{b}^{2 a+1}\right) \approx B P^{*}\left(R P_{b}^{2 a}\right) \oplus \Sigma^{2 a+1} B P^{*}$.

$B P^{*}\left(R P_{2 b}^{a}\right) \approx B P^{*}\left(R P_{2 b+1}^{a}\right) \oplus \Sigma^{2 b} B P^{*}$.

The Landweber short exact sequence

$$
\begin{gathered}
0 \longrightarrow B P^{*}\left(R P_{b}^{a}\right) \otimes_{B P^{*}} B P^{*}\left(R P_{d}^{c}\right) \longrightarrow B P^{*}\left(R P_{b}^{a} \wedge R P_{d}^{c}\right) \\
\longrightarrow \operatorname{Tor}^{B P^{*}}\left(B P^{*}\left(R P_{b}^{a}\right), B P^{*}\left(R P_{d}^{c}\right)\right) \longrightarrow 0
\end{gathered}
$$

always splits.

Combined, this allows us to compute the $B P$ cohomology of any such product. Since there is no $v_{i}$ torsion for $i \geqslant 2$ we can really use this for any $B P\langle n\rangle^{*}(-)$ for $n>0$ and, of course, we can always get $E(n)^{*}(-)$ from $B P^{*}(-)$ by just tensoring, $E(n)^{*}(-) \approx E(n)^{*} \otimes_{B P^{*}} B P^{*}(-),[\mathbf{J W 7 3}$, Remark 5.13, p. 347]. In particular, what is used in [KWa, $\mathbf{K W b}]$ is the case of $E(2)$. Since there is no $v_{i}$ torsion for $i \geqslant 2$, we know that $B P\langle n\rangle^{*}(-)$ injects to $E(n)^{*}(-), n>1$, for these spaces.

$B P$ homology computations can be done independently purely algebraically, mimicking the way they are done in cohomology, or, one can just use S-duality where 
we have from [Ati61] that the S-dual of $R P_{b}^{a}$ is $R P_{2^{k}-a-1}^{2^{k}-b-1}$ (for some large $k$ ). Our computations for cohomology are immediate for homology.

For $B P_{*}\left(R P^{2 n}\right)$ we have generators $\beta_{i} \in B P_{2 i-1}\left(R P^{2 n}\right)$ for $0<i \leqslant n$. The basic facts for homology are collected as a theorem:

Theorem 1.4. The Landweber short exact sequence (1) for $B P_{*}\left(R P_{b}^{a} \wedge R P_{d}^{c}\right)$ always splits. The map to Tor decreases degree by 1.

Any top and bottom 'integral' cells split off in BP homology.

Let $m \leqslant n$; then

$$
\operatorname{Tor}^{B P_{*}}\left(B P_{*}\left(R P^{2 m}\right), B P_{*}\left(R P^{2 n}\right)\right) \approx \Sigma B P_{*}\left(R P^{2 m}\right) .
$$

A 2-adic basis for $B P_{*}\left(R P^{2 k}\right)$ is given by all $v \beta_{i}$ with $0<i \leqslant k$, and where $v$ ranges over a $\mathbb{Z}_{(2)}$-basis of $B P_{*}$.

$A$ 2-adic basis for $B P_{*}\left(R P^{2 m}\right) \otimes_{B P_{*}} B P_{*}\left(R P^{2 n}\right)$ is given by all $v \beta_{i} \otimes \beta_{j}$ with $0<i \leqslant m, 0<j \leqslant n$, and where $v$ ranges over a $\mathbb{Z}_{(2)}$-basis of $B P_{*}$ when $j=n$ and over a $\mathbb{Z}_{(2)}$-basis of $\mathbb{Z}_{(2)}\left[v_{2}, v_{3}, \ldots\right]$ when $j<n$.

The splittings associated with the 'integral' cells are a consequence of Don Davis's result from [Dav78] that proves they really do split off topologically when smashed with $B P$.

We can generalize these results to the case of $L(e)_{b}^{a} \wedge R P_{d}^{c}$ where $L(e)_{b}^{a}$ is the truncated lens space for $2^{e}$ (when $e=1$ it is just the case $R P_{b}^{a}$ we have already described). Let $\alpha_{i} \in B P_{2 i-1}\left(L(e)^{a}\right)$ to distinguish it from our $\beta_{i}$ and let $x_{e} \in B P^{2}\left(L(e)^{a}\right)$ come from $B P^{*}\left(C P^{\infty}\right)$.

Some facts we'll need (let $v_{0}=2$ ):

Theorem 1.5. A 2-adic basis for $B P^{*}\left(L(e)^{2 k}\right)$ is given by all $v_{0}^{j} v x_{e}^{i}$ with $0 \leqslant j<e$, $0<i \leqslant k$, and where $v$ ranges over a $\mathbb{Z}_{(2)}$-basis of $B P^{*}$.

Let $n \geqslant m+e-1$ and $m>1$; then a 2-adic basis for $B P^{*}\left(L(e)^{2 m}\right) \otimes_{B P^{*}}$ $B P^{*}\left(R P^{2 n}\right)$ is given by all $v x_{e}^{i} \otimes x^{j}$ with $0<i \leqslant m, 0<j \leqslant e$, and where $v$ ranges over a $\mathbb{Z}_{(2)}$-basis of $B P^{*}$, together with all $v_{1}^{\ell} v x_{e}^{i} \otimes x^{j}$ with $0<i \leqslant m, e<j \leqslant n$, $0 \leqslant \ell<e$, and where $v$ ranges over a $\mathbb{Z}_{(2)}$-basis of $\mathbb{Z}_{(2)}\left[v_{2}, v_{3}, \ldots\right]$.

$A$ 2-adic basis for $B P_{*}\left(L(e)^{2 k}\right)$ is given by all $v_{0}^{j} v \alpha_{i}$ with $0 \leqslant j<e, 0<i \leqslant k$, and where $v$ ranges over a $\mathbb{Z}_{(2)}$-basis of $B P^{*}$.

Let $n \geqslant m+e-1$ and $m>1$; then a 2-adic basis for $B P_{*}\left(L(e)^{2 m}\right) \otimes_{B P_{*}}$ $B P_{*}\left(R P^{2 n}\right)$ is given by all $v \alpha_{i} \otimes \beta_{j}$ with $0<i \leqslant m, n-e<j \leqslant n$, and where $v$ ranges over a $\mathbb{Z}_{(2)}$-basis of $B P_{*}$, together with all $v_{1}^{\ell} v \alpha_{i} \otimes \beta_{j}$ with $0<i \leqslant m, 0<j \leqslant$ $n-e, 0 \leqslant \ell<e$, and where $v$ ranges over a $\mathbb{Z}_{(2)}$-basis of $\mathbb{Z}_{(2)}\left[v_{2}, v_{3}, \ldots\right]$.

The Landweber short exact sequence (1) for $L(e)_{b}^{a} \wedge R P_{d}^{c}$ always splits.

Similar identities to those in Theorem 1.3 hold for $B P^{*}\left(L(e)_{b}^{a}\right)$ and $B P_{*}\left(L(e)_{b}^{a}\right)$.

Remark 1.6. When $m=1$, this is just the mod $2^{e} B P$-(co)homology of $R P^{2 n}$. The proof works here for $n>e$ as well and the result is as stated. The case of $n \leqslant e$ is even easier.

This allows us to compute the $B P$ (co)homology of $L(e)_{b}^{a} \wedge R P_{d}^{c}$ with some restrictions just as we did in the $e=1$ case with one significant difference: we have lost our elegance when describing our Tor term. Consequently we bury our description in 
the section with the proofs. We will also describe why we need the extra bit in our inequality.

In order to prove this we rely on the result of G. Nakos [Nak85], see also [Col85, Gon03], that says that the annihilator ideal for the bottom class $\alpha_{1} \otimes \beta_{1}$ in $B P_{*}\left(B \mathbb{Z} /\left(2^{e}\right) \wedge B \mathbb{Z} /(2)\right)$ is $\left(2, v_{1}^{e}\right)$. The $B P$ cohomology has been understood for a long time $[$ Lan70].

We also compute $B P_{*}\left(L(e)^{2 m} \wedge R P^{2}\right) . R P^{2}$ is just the mod 2 Moore space, and when $m \geqslant 2^{e}$, we get an annihilator ideal of $\left(2, v_{1}^{2^{e}-1}\right)$. As $n$ goes from 1 to $m+e-1$, this annihilator ideal must grow from $\left(2, v_{1}^{2^{e}-1}\right)$ to $\left(2, v_{1}^{e}\right)$. Things get quite complex in this range.

Part of this work was completed during a sabbatical visit of the first author to the University of Rochester. The visit was financially supported by a grant of Professor Douglas C. Ravenel and CONACyT grant 54987-E. It is a pleasure to thank Professor Ravenel for his kind help and academic motivation during this visit.

\section{Proofs of Theorems 1.1 and $\mathbf{1 . 2}$}

We recall the formal group law for Brown-Peterson cohomology, $x+{ }_{F} y$ and the corresponding 2-series (where $v_{0}=2$ ):

$$
x+{ }_{F} x=[2](x)=\sum_{i \geqslant 0} a_{i} x^{i+1}=\sum_{n \geqslant 0}{ }_{F} v_{n} x^{2^{n}} .
$$

Note that this immediately implies that $a_{0}=2$ and $a_{1}=v_{1}$ (for a general reference see [Wil80]; we are using Araki's generators here [Ara73]).

The maps

$$
R P^{\infty} \longrightarrow C P^{\infty} \stackrel{2}{\longrightarrow} C P^{\infty}
$$

give us a short exact sequence

$$
B P^{*}\left(R P^{\infty}\right) \longleftarrow B P^{*}\left(C P^{\infty}\right) \stackrel{2^{*}}{\longleftarrow} B P^{*}\left(C P^{\infty}\right) .
$$

In terms of unreduced cohomology, this corresponds to the short exact sequence of algebras

$$
B P^{*}[[x]] /([2](x)) \longleftarrow B P^{*}[[x]] \stackrel{2^{*}}{\longleftarrow} B P^{*}[[x]] .
$$

The Atiyah-Hirzebruch spectral sequence for $B P^{*}\left(R P^{\infty}\right)$ collapses because it is even degree and the 2-series shows how to solve all the extension problems. The same is true for $B P^{*}\left(R P^{2 n}\right)$ and now we inherit, from $C P^{\infty}$ and $C P^{n}$,

$$
B P^{*}\left(R P^{2 n}\right) \approx B P^{*}[x] /\left([2](x), x^{n+1}\right) .
$$

The Atiyah-Hirzebruch spectral sequence gives our 2-adic basis for $B P^{*}\left(R P^{2 n}\right)$ and we see that our relations are given by $\sum_{i \geqslant 0} a_{i} x^{j+i}$. (In homology they are given by $\sum_{i \geqslant 0} a_{i} \beta_{j-i}$.)

We can show how to reduce any element in the tensor product, $B P^{*}\left(R P^{2 m}\right) \otimes_{B P^{*}}$ $B P^{*}\left(R P^{2 n}\right)$, with $m \leqslant n$ to the 2 -adic basis of the Theorem 1.1. We need to filter the tensor product to make this easy. First we filter on the sum, $i+j$, for $x^{i} \otimes x^{j}$. Next, if $x^{a} \otimes x^{b}$ has $a+b=i+j$, we let it have higher filtration if $b<j$. We set up 
an algorithm for reduction. If we have an element that is divisible by 2 , i.e. if we have a $2 x^{i} \otimes x^{j}$, we replace the $2 x$ in $2 x x^{i-1}$ using the 2 -series. All terms are of a higher filtration. If we have a $v_{1} x^{i} \otimes x^{j}$ with $j>1$, we replace the $v_{1} x^{2}=a_{1} x^{2}$ in $v_{1} x^{2} x^{j-2}$ using the 2-series. All of the terms with $a_{i}, i>1$ will be of higher filtration, but we will be left with $-2 x^{i} \otimes x^{j-1}$. We can now replace the $2 x$ in $2 x x^{i-1}$ using the 2 -series, and all of our terms will be of higher filtration.

This shows that we can reduce all terms in the tensor product to the 2-adic basis in our first theorem. It does not prove they form a basis, though, so beware. The tensor product could be smaller than this until we prove otherwise. We have proven that this is the largest the tensor product could possibly be, though.

The Landweber short exact sequence applies to any $X$ and $Y$ where $B P_{*}(X) \rightarrow$ $H_{*}(X)$ is surjective, or, in other words, the Atiyah-Hirzebruch spectral sequence collapses. In such a case there is a free $B P_{*}$ resolution:

$$
0 \longrightarrow A_{1} \longrightarrow A_{0} \longrightarrow B P_{*}(X) \longrightarrow 0 .
$$

To see much more of this type of thing, go to [CS69].

The Landweber short exact sequence now comes from this resolution by tensoring with $B P_{*}(Y)$. The tensor product is just the cokernel of

$$
A_{1} \otimes_{B P_{*}} B P_{*}(Y) \longrightarrow A_{0} \otimes_{B P_{*}} B P_{*}(Y)
$$

and the Tor term is the kernel.

For finite complexes, Spanier-Whitehead duality allows us to switch to cohomology (a Künneth Spectral Sequence argument can alternatively be used, observing that in either homology or cohomology, the factors we are interested in have homological dimension 1, so the whole spectral sequence collapses to the standard short exact sequence) and, in the case of $R P^{2 m}$, we can write down the resolution explicitly. We let $A_{0}$ be free on generators $d_{i}, 0<i \leqslant m$ of degree $2 i$. The map $A_{0} \rightarrow B P^{*}\left(R P^{2 m}\right)$ is given by $d_{i} \rightarrow x^{i} . A_{1}$ is free on $c_{i}, 0<i \leqslant m$ of degree $2 i$ and the map $\partial: A_{1} \rightarrow A_{0}$ is given by $\partial\left(c_{i}\right)=\sum_{j \geqslant 0} a_{j} d_{i+j}$.

Our Tor of interest is the kernel of:

$$
A_{1} \otimes_{B P^{*}} B P^{*}\left(R P^{2 n}\right) \longrightarrow A_{0} \otimes_{B P^{*}} B P^{*}\left(R P^{2 n}\right) .
$$

We start by finding an injection

$$
\Sigma^{2 n} B P^{*}\left(R P^{2 m}\right) \longrightarrow A_{1} \otimes_{B P^{*}} B P^{*}\left(R P^{2 n}\right) .
$$

Let

$$
\Sigma^{2 n} x^{j} \longrightarrow \sum_{i \geqslant 0} c_{i+j} \otimes x^{n-i}
$$

First we have to show this is well defined by showing that the relations go to zero:

$$
\Sigma^{2 n}\left(\sum_{j \geqslant 0} a_{j} x^{k+j}\right) \longrightarrow \sum_{j \geqslant 0} a_{j} \sum_{i \geqslant 0} c_{i+j+k} \otimes x^{n-i} .
$$

Fix $i+j=b$ and look at the coefficient of $c_{b+k}$. We have

$$
\sum_{i+j=b} a_{j} x^{n-i}=\sum_{j=0}^{b} a_{j} x^{n-b+j}=0
$$


To see that this map is an injection, all we have to do is map to the quotient of $A_{1} \otimes B P^{*}\left(R P^{2 n}\right)$ obtained by setting all $c_{i}=0$ except for $c_{m}$. This gives us a map

$$
\Sigma^{2 n} B P^{*}\left(R P^{2 m}\right) \longrightarrow \Sigma^{2 m} B P^{*}\left(R P^{2 n}\right)
$$

that takes $\Sigma^{2 n} x^{j}$ to $\Sigma^{2 m} x^{n-m+j}$. This injects on the 2-adic basis.

Our next step is to show our image is in the kernel. We have:

$$
\Sigma^{2 n} x^{j} \longrightarrow \sum_{i \geqslant 0} c_{i+j} \otimes x^{n-i} \longrightarrow \sum_{i \geqslant 0} \sum_{k \geqslant 0} a_{k} d_{i+j+k} \otimes x^{n-i} .
$$

Again, fix $i+k=b$ and find the coefficient of $d_{b+j}$ :

$$
\sum_{i+k=b} a_{k} x^{n-i}=\sum_{k=0}^{b} a_{k} x^{n-b+k}=0 .
$$

So far we have shown that the tensor product can be no bigger than Theorem 1.1 states and that the Tor term in Theorem 1.2 can be no smaller than what we have already found is in the kernel.

Each of the $A_{i} \otimes B P^{*}\left(R P^{2 n}\right)$ is a finite abelian 2-group. Furthermore, the $i=0$ and 1 groups are isomorphic. Thus the kernel and the cokernel must be exactly the same size in each degree. Thus if the elements we have found so far in the kernel are exactly the same size as our proposed tensor product, then we are done because our tensor product cannot be smaller than what we already know is in the kernel. This is now just a simple counting argument.

The 2-adic bases for both what we have already in the kernel and what we propose for the tensor product have $v_{n}$ injective modulo $\left(v_{n+1}, v_{n+2}, \ldots\right)$ for $n>1$, so we can ignore all of the $v_{i}, i>1$ in our counting argument. We just give a 1-1 correspondence for what is left. For $0<j \leqslant m$, map $\Sigma^{2 n} v_{1}^{i} x^{j}$ to $x^{j} \otimes x^{n-i}$ for $0 \leqslant i<n$ and to $v_{1}^{i-n+1} x^{j} \otimes x$ for $i \geqslant n$.

We must take care of the naturality, since that is one of the motivating factors for this paper. If $k<m$ and we have $R P^{2 k} \rightarrow R P^{2 m}$, we get the obvious surjection of resolutions $A_{i}^{m} \rightarrow A_{i}^{k}$ and the map of $\Sigma^{2 n} x^{j}$ is preserved except that it is zero when $j>k$. This shows the first part of the naturality.

If $m<n$ and we map $R P^{2 n-2}$ to $R P^{2 n}$, the map of $\Sigma^{2 n} x^{j}$ to $A_{1} \otimes B P^{*}\left(R P^{2 n}\right)$ to $A_{1} \otimes B P^{*}\left(R P^{2 n-2}\right)$ goes

$$
\Sigma^{2 n} x^{j} \longrightarrow \sum_{i=0}^{m-j} c_{i+j} \otimes x^{n-i} \longrightarrow \sum_{i=1}^{m-j} c_{i+j} \otimes x^{n-i} \quad\left(x^{n}=0 \text { here }\right)
$$

If we go

$$
\Sigma^{2 n} x^{j} \longrightarrow \Sigma^{2 n-2} x^{j+1} \longrightarrow \sum_{i=0}^{m-j-1} c_{i+j+1} \otimes x^{n-1-i},
$$

we see we have the same thing and this shows the second part of the naturality on Tor.

It is elementary that $B P^{*}\left(R P^{2 n+1}\right) \approx B P^{*}\left(R P^{2 n}\right) \oplus B P^{*}\left(S^{2 n+1}\right)$, so the tensor product and Tor can be computed from this fact. 
The only thing left to do is show that there can be no extension problems, i.e. that Landweber's short exact sequence splits. This problem is solved in $B P$ homology using the result from $[\mathbf{D a v 7 8}]$ that says

$$
B P \wedge R P_{b}^{2 a+1} \simeq B P \wedge R P_{b}^{2 a} \vee \Sigma^{2 a+1} B P
$$

and

$$
B P \wedge R P_{2 b}^{a} \simeq B P \wedge R P_{2 b+1}^{a} \vee \Sigma^{2 b} B P .
$$

Since this splits topologically, there can be no algebra extensions. By S-duality the same is true for cohomology.

It should be noted that the above splittings are proved in [Dav78] for spaces with only one integral cell, but having one at each end presents no serious problem: Davis' topological argument relies solely on knowing the surjectivity in BP-homology of the pinch map $R P_{2 b}^{2 a+1} \rightarrow S^{2 a+1}$. But this is assured by the corresponding situation for $R P_{2 b-1}^{2 a+1} \rightarrow S^{2 a+1}$.

\section{Proof of Theorem $\mathbf{1 . 5}$}

To describe $B P^{*}\left(L(e)^{2 k}\right)$, we need to take the formal group sum of $x_{e} 2^{e}$ times to get

$$
\left[2^{e}\right]\left(x_{e}\right)=\sum_{i \geqslant 0} a_{i, e} x_{e}^{i+1} .
$$

We need some facts about these elements:

Lemma 3.1 ([Gon01]). $a_{s, e}$ is divisible by $2^{\mu(s)}$, where

$$
\mu(s)=\sum_{0 \leqslant i<e} b_{i}(e-i)
$$

and $s+1=\sum 2^{i} b_{i}$ is the 2-adic expression of $s+1$.

All we need is the fact that $2^{e-s+1}$ divides $a_{s, e}$ for $1<s \leqslant e+1$. Notice however that this is not the case for $s \in\{0,1\}: a_{0, e}=2^{e}$ and, up to units, $a_{1, e}=2^{e-1} v_{1}$ (precisely, $\left.a_{1, e}=2^{e-1}\left(2^{e}-1\right) v_{1}\right)$.

With diagrams similar to those in the $e=1$ case, we have

$$
B P^{*}\left(L(e)^{2 n}\right) \approx B P^{*}\left[x_{e}\right] /\left(\left[2^{e}\right]\left(x_{e}\right), x_{e}^{n+1}\right) .
$$

The Atiyah-Hirzebruch spectral sequence for $B P^{*}(L(e))$ collapses because it is even degree and the $2^{e}$-series shows how to solve all the extension problems. The same is true for $B P^{*}\left(L(e)^{2 n}\right)$.

The Atiyah-Hirzebruch spectral sequence gives our 2-adic basis for $B P^{*}\left(L(e)^{2 k}\right)$ and we see that our relations are given by $\sum_{i \geqslant 0} a_{i, e} x_{e}^{j+i}$. (In homology they are given by $\sum_{i \geqslant 0} a_{i, e} \alpha_{j-i}$.)

It does not matter whether we work with homology or cohomology, as they are really equivalent. This time we will work with homology.

We begin with our Landweber short exact sequence just as before, but this time we resolve $B P_{*}\left(L(e)^{2 m}\right)$. As before, we take free $A_{i}^{e}$ on the same generators with a 
shift of degree by 1 , but now the maps are different: The map $A_{0}^{e} \rightarrow B P_{*}\left(L(e)^{2 m}\right)$ is given by $d_{i} \rightarrow \alpha_{i}$ and the map $\partial: A_{1}^{e} \rightarrow A_{0}^{e}$ is given by $\partial\left(c_{i}\right)=\sum_{j \geqslant 0} a_{j, e} d_{i-j}$.

Our Tor is the kernel of

$$
A_{1}^{e} \otimes_{B P_{*}} B P_{*}\left(R P^{2 n}\right) \longrightarrow A_{0}^{e} \otimes_{B P_{*}} B P_{*}\left(R P^{2 n}\right)
$$

and the tensor product is the cokernel.

Computing this kernel and cokernel is significantly different from what was done before. We can't do it directly but need to set up a spectral sequence to help us do it. This is because we have no analogue to the explicit computation of the kernel that we had before.

We define a decreasing filtration on our short chain complex: For $z=c$ or $d$ :

$$
F\left(z_{i} \otimes \beta_{j}\right)=2 i+j, \text { and } F\left(B P_{*}\right)=0 .
$$

A 2 -adic basis for our spectral sequence is given as a free $B P_{*} /(2)$ module on generators $z_{i} \otimes \beta_{j}$ with $0<i \leqslant m$ and $0<j \leqslant n$.

We compute the first differential using:

$$
\partial\left(c_{i} \otimes \beta_{j}\right)=2^{e} d_{i} \otimes \beta_{j}+a_{1, e} d_{i-1} \otimes \beta_{j}+a_{2, e} d_{i-2} \otimes \beta_{j}+\cdots .
$$

All terms with 2 in them can be eliminated by using the 2 -series on the right hand factor. We see that the summand $a_{s, e} d_{i-s} \otimes \beta_{j}$ has filtration

- at most $2(i-1)+j-e+1$, when $s=1$;

- at most $2(i-s)+j-e+s-1$, when $1<s \leqslant e+1$;

- at most $2(i-e-2)+j$, when $s \geqslant e+2$.

However, the leading-filtration term from the case $s=0$ is given by $v_{1}^{e} d_{i} \otimes \beta_{j-e}$, which has a larger filtration than that observed in any of the three cases above. We have thus proved:

Proposition 3.2. For $n>e$, the first non-trivial differential in the spectral sequence under consideration is $\delta_{e}\left(c_{i} \otimes \beta_{j}\right)=v_{1}^{e} d_{i} \otimes \beta_{j-e}$.

Corollary 3.3. For any $n>0$, the $E_{e+1}$ term of our spectral sequence is described as follows:

1. In homological degree 1 , it is a free $B P_{*} /(2)$-module on generators $c_{i} \otimes \beta_{j}$ satisfying $0<i \leqslant m$ and $0<j \leqslant \min \{n, e\}$.

2. In homological degree 0 , it is free over $B P_{*} /(2)$ on $d_{i} \otimes \beta_{j}$ with $n-e<j \leqslant n$, and over $B P_{*} /\left(2, v_{1}^{e}\right)$ on $d_{i} \otimes \beta_{j}$ with $0<j \leqslant n-e$.

Proposition 3.4. For $n \geqslant m+e-1$ and $m>1$ the spectral sequence collapses after the $\delta_{e}$-differential in Proposition 3.2. In particular, Corollary 3.3 describes a filtered version of tensor, Tor, and $B P_{*}\left(L(e)^{2 m} \wedge R P^{2 n}\right)$.

Remark 3.5. The same description and proof work when $m=1$, provided $n>e$. On the other hand, when $n \leqslant e$, multiplication by $2^{e}$ is trivial on the $B P$-(co)homology of $R P^{2 n}$, so that the considerations above Proposition 3.2 show that the first non-trivial differential $\delta_{t}$ (if any) will hold for $t>e$. As an extreme case of this situation, we note that the whole spectral sequence collapses for $m=1$ and $n \leqslant e$. 
In our proof we will need to use the Smith homomorphism $\kappa: A_{i}^{e} \rightarrow A_{i}^{e}$ determined by $\kappa\left(z_{i}\right)=z_{i-1}$. Since this works on the quotient $B P_{*}\left(L(e)^{2 m}\right)$ and also for $e=1$, we have $\kappa_{r, s}=\kappa^{r} \otimes \kappa^{s}: A_{i}^{e} \otimes B P_{*} P^{2 n} \rightarrow A_{i}^{e} \otimes B P_{*} P^{2 n}$ is compatible with the filtered chain complex giving our spectral sequence and therefore produces a spectral sequence (graded) endomorphism.

Proof. We proceed by contradiction. Assume that one of the generators $c_{i} \otimes \beta_{j}$ in Corollary 3.3 (1) supports a non-trivial differential

$$
\delta_{m}\left(c_{i} \otimes \beta_{j}\right)=c d_{r} \otimes \beta_{s}+\cdots .
$$

Choose $m$ minimal with $c d_{r} \otimes \beta_{s}$ non-zero. Of all the possible $(r, s)$ pairs in this filtration, we choose the one with $r+s$ maximal; i.e. with $s$ maximal. Using the spectral sequence morphism $\kappa_{r-1, s-1}$, we can pull down (3) to a differential

$$
\delta_{m}\left(c_{i-r+1} \otimes \beta_{j-s+1}\right)=c d_{1} \otimes \beta_{1} .
$$

From [Nak85, Col85, Gon03], we know that $c$ must be zero in $B P_{*} /\left(2, v_{1}^{e}\right)$ because the annihilator ideal of $\alpha_{1} \otimes \beta_{1}$ cannot be bigger than $\left(2, v_{1}^{e}\right)$.

We know that the only elements left that could have a differential are the $c_{i} \otimes \beta_{j}$ with $0<j \leqslant e$ and we know that the target must be some $c d_{r} \otimes \beta_{s}+\cdots$ with $n-e<$ $s \leqslant n$ and $c=v_{1}^{e} a$. Thus the degree of the target must be at least $2 e+2 r-1+$ $2(n-e)+1=2 n+2 r$. The degree of the source is at most $2 i-1+2 e-1$. There can be no differential if the maximum possible degree of a potential source is less than the minimum possible degree of a potential target; i.e. $i+e-1<n+r$. Since $i-r$ must be less than or equal to $m-1$, this follows from $m+e-2<n$, which was our assumption.

The only thing left to do is show that there can be no tensor-Tor extension problems in a general product $L(e)_{b}^{a} \wedge R P_{d}^{c}$ involving integral cells; i.e. that Landweber's short exact sequence splits. As in Section 2, this problem is solved in $B P$ homology using the same techniques for lens spaces that [Dav78] uses for truncated projective spaces. The $B P$ cohomology situation is handled using the fact that truncated lens spaces have S-duals just like the real projective spaces [Kob94, Lemma 2.2]. Of course we have plenty of unsolved extension problems anyway.

\section{Two examples}

Example 4.1. $B P_{*}\left(R P^{2}\right)=B P_{*} /(2)$ on $\beta_{1}$. The first term of the $\left[2^{e}\right]\left(x_{e}\right)$ series that is non-zero mod 2 is $a_{2^{e}-1, e}$ and it is $v_{1}^{2^{e}-1} \bmod 2$ (see [Gon01]). The first and only differential in our spectral sequence comes from the chain map

$$
c_{i} \otimes \beta_{1} \longrightarrow v_{1}^{2^{e}-1} d_{i-2^{e}+1} \otimes \beta_{1}+\text { low }
$$

where low stands for "lower filtration elements." This means that the only differential in the $n=1$ homology version of the spectral sequence is given by $c_{i} \otimes \beta_{1} \mapsto$ $v_{1}^{2^{e}-1} d_{i-2^{e}+1} \otimes \beta_{1}$. The tensor and Tor products in this case $(n=1)$ can now be read off from the resulting $E_{\infty}$ term. For instance, when $m<2^{e}, \partial=0$, so that tensor and Tor products are both isomorphic to $A_{i} \otimes B P_{*}\left(R P^{2}\right)$. However, when $m \geqslant 2^{e}$, the Tor product has a $B P_{*} /(2)$ free 2 -adic basis given by the elements $c_{i} \otimes \beta_{1}$, for $0<i<2^{e}$, 
whereas the tensor factor has a graded associated object generated by all $d_{i} \otimes \beta_{1}$ $(0<i \leqslant m)$, free over $B P_{*} /(2)$ when $m-2^{e}+1<i \leqslant m$ and over $B P_{*} /\left(2, v_{1}^{2^{e}-1}\right)$ when $0<i \leqslant m-2^{e}+1$. In any case, since the bottom class $\alpha_{1} \otimes \beta_{1}$ in the tensor product is the lowest possible filtration generator, we see that its $B P_{*}$-annihilator ideal does not depend on whether we consider this class as an element in the actual tensor product or as an element in the associated graded $E_{\infty}$ term. For instance, when $m \geqslant 2^{e}$, this common annihilator ideal is generated by 2 and $v_{1}^{2^{e}-1}$. As $n$ increases from 1 to $m+e-1$, the corresponding ideal increases to $\left(2, v_{1}^{e}\right)$, which is the (constant) annihilator ideal of $\alpha_{1} \otimes \beta_{1}$ for all $n \geqslant m+e-1$.

Example 4.2. Consider the case with $e=2$ and $m=n=3$. The first round of differentials (identified in Proposition 3.2) are given by $c_{i} \otimes \beta_{3} \mapsto v_{1}^{2} d_{i} \otimes \beta_{1}$ for $i=1,2,3$. However, a straightforward calculation shows that in our chain complex there holds the relation $\partial\left(c_{3} \otimes \beta_{2}+c_{2} \otimes \beta_{3}\right)=v_{1}^{2} d_{1} \otimes \beta_{2}$. This means that the spectral sequence has the extra $\delta_{4}$-differential $c_{3} \otimes \beta_{2} \mapsto v_{1}^{2} d_{1} \otimes \beta_{2}$. It can easily be verified that all other elements left in homological degree 1 are in fact permanent cycles, so that the spectral sequence collapses from its fifth stage.

\section{References}

[Ara73] S. Araki, Typical formal groups in complex cobordism and $K$-theory, Lectures in Mathematics, Department of Mathematics, Kyoto University, No. 6, Kinokuniya Book-Store Co., Ltd, Tokyo, 1973.

[Ati61] M.F. Atiyah, Thom complexes, Proc. London Math. Soc. (3) 11 (1961), 291-310.

[CF64] P. E. Conner and E. E. Floyd, Differentiable periodic maps, Ergebnisse der Mathematik und ihrer Grenzgebiete, N. F., Band 33, Academic Press Inc., Publishers, New York, 1964.

[Col85] R.A. Coley, Projective dimension of $B P_{*} B G$ for finite groups, $\mathrm{PhD}$ thesis, Massachusetts Institute of Technology, 1985.

[CS69] P.E. Conner and L. Smith, On the complex bordism of finite complexes, Inst. Hautes Études Sci. Publ. Math. 37 (1969), 117-221.

[Dav84] D.M. Davis, A strong nonimmersion theorem for real projective spaces, Ann. of Math. (2) 120 (1984), 517-528.

[Dav78] D.M. Davis, The BP-coaction for projective spaces, Canad. J. Math. 30 (1978), 45-53.

[Gon01] J. González, 2-divisibility in the Brown-Peterson $\left[2^{k}\right]$-series, J. Pure Appl. Algebra 157 (2001), 57-68.

[Gon03] J. González, A generalized Conner-Floyd conjecture and the immersion problem for low 2-torsion lens spaces, Topology 42 (2003), 907-927.

[JW73] D. C. Johnson and W. S. Wilson, Projective dimension and BrownPeterson homology, Topology 12 (1973), 327-353.

[JW85] D. C. Johnson and W. S. Wilson, The Brown-Peterson homology of elementary p-groups, Amer. J. Math. 107 (1985), 427-454. 
[Kob94] T. Kobayashi, Stable homotopy types of stunted lens spaces $\bmod p^{r}$, Mem. Fac. Sci. Kochi Univ. Ser. A Math. Math. 15 (1994), 9-14.

[KWa] N. Kitchloo and W.S. Wilson, The second real Johnson-Wilson theory and non-immersions of $R P^{n}$, Homology, Homotopy Appl. 10(3) (2008), $223-268$.

[KWb] N. Kitchloo and W.S. Wilson, The second real Johnson-Wilson theory and non-immersions of $R P^{n}$, Part 2, Homology, Homotopy Appl. 10(3) (2008), 269-290.

[Lan66] P.S. Landweber, Künneth formulas for bordism theories, Trans. Amer. Math. Soc. 121 (1966), 242-256.

[Lan70] P. S. Landweber, Coherence, flatness and cobordism of classifying spaces, In Proc. Advanced Study Inst. on Algebraic Topology, pages 256-269, Aarhus, 1970.

[Nak85] G. Nakos, On the Brown-Peterson homology of certain classifying spaces, PhD thesis, Johns Hopkins University, 1985.

[Wil80] W.S. Wilson, Brown-Peterson Homology: an Introduction and Sampler, CBMS Regional Conference Series in Mathematics, No. 48, AMS, Providence, RI, 1980.

Jesús González jesus@math.cinvestav.mx

Departamento de Matemáticas, Centro de Investigación y de Estudios Avanzados del IPN, A. P. 14740, México City 07000, MÉXICO

W. Stephen Wilson wsw@math.jhu.edu

Department of Mathematics, Johns Hopkins University, Baltimore, MD 21218, USA 